\title{
Protective and Risk Factors in Multiple Sclerosis
}

\section{Hosein Nouri ${ }^{1}$, Homayoun Tabesh ${ }^{2}$, Masih Saboori ${ }^{1,2}$, Karim Sohrabi ${ }^{1}$, Foad Mirhosseini ${ }^{1}$, Zahra Hosseinzadeh Anvar ${ }^{1}$, Mostafa Sharifkhah', Saman Hosseinzadeh Anvar', Vahid Shaygannejad ${ }^{1 *}$}

\author{
${ }^{1}$ Isfahan Neurosciences Research Center, Alzahra Research Institute, Isfahan University of Medical Sciences, Isfahan, Iran \\ ${ }^{2}$ Department of Neurosurgery, Isfahan University of Medical Sciences, Isfahan, Iran
}

Corresponding Author: Vahid Shaygannejad, Neurologist, Professor, Isfahan Neurosciences Research Center, Alzahra Research Institute, Isfahan University of Medical Sciences, Isfahan, Iran. Email: Shaygannejad@med.mui.ac.ir

Received December 24, 2018; Accepted May 21, 2019; Online Published June 15, 2019

\begin{abstract}
Several factors are involved in susceptibility to multiple sclerosis (MS) and alterations in disease activity. A full understanding regarding the mechanism which causes the disease is still lacking. Several factors have been considered to be associated, positively or negatively, with the onset, course, progression, and relapse rates of MS. However, the level of consensus on each factor is different. These factors are either genetic (including polymorphisms in human leukocyte antigen, microRNAs genes, etc) or environmental (such as serum levels of circulating vitamin D, helicobacter pylori, Epstein-Barr virus, human cytomegalovirus, human herpes virus type 6, human endogenous retrovirus infections, and latitude). Vitamin D sufficiency is a major protective factor on which there is a decisive consensus and has known interactions with many factors affecting MS onset/severity. Vitamin D therapy is speculated to exert beneficial effects on patients, the efficacy of which, along with the etiology of MS, is reviewed in this article.

Keywords: Multiple Sclerosis, Vitamin D, Therapeutics, Risk Factors, Autoimmunity

Citation: Nouri H, Tabesh H, Saboori M, et al. Protective and risk factors in multiple sclerosis. Int J Med Rev. 2019;6(2):51-58. doi:10.29252/ IJMR-060205.
\end{abstract}

\begin{abstract}
Introduction
Multiple sclerosis (MS) is a chronic inflammatory, demyelinating disease of the central nervous system (CNS). Damage to myelin proteins occurs during MS progression and relapse. Over 2 million people suffer from the consequences of pathological mechanisms which MS involves, monocular visual loss, limb weakness/sensory loss, diplopia (double vision) and ataxia resulting from optic neuritis, transverse myelitis, brainstem dysfunction, and cerebellar lesions being among them. ${ }^{1}$ Based on the disease course, MS patients can be divided into four main types: I) Relapsing-remitting MS (RRMS), which is by far the most common type, affecting about $85 \%$ of MS patients; II) Secondary progressive MS (SPMS), which may progress in patients with relapsingremitting MS; III) Primary progressive MS (PPMS); and IV) Progressive-relapsing MS (PRMS). ${ }^{2}$ A recently detected subtype of the disease described as "myelocortical MS" argues against the idea that neural degeneration necessarily occurs following demyelination. In patients with myelocortical MS, demyelinated lesions in cerebral white matter were not observed. Similar to typical MS courses, however, the mean cortical neuronal densities were significantly lower than those of the healthy controls. ${ }^{3}$ These new findings will further improve the diagnoses and treatments utilized in MS patients.
\end{abstract}

\section{Genetic Factors}

Human Leukocyte Antigen and microRNAs Genes

The human leukocyte antigen (HLA)-DRB1 gene explains up to $10.5 \%$ of the genetic variance underlying risk. ${ }^{4}$ HLA-DRB1*15:01 allele has the strongest association with increased MS risk among genetic risk factors and has been consistently associated with MS susceptibility in approximately all populations studied, pointing to a specific antigen presentation as the pathogenic mechanism.

Based on previous studies, the association between MS and the HLA-DR15 haplotype (HLA-DRB1*15:01 allele and the alleles in linkage disequilibrium with it; HLA-DQB1*0602 and HLA-DQA1*0102) has been shown throughout both European and non-European populations, ${ }^{5}$ except in Sardinian individuals, among whom MS susceptibility has been reported to be associated with HLA-DR4 (DRB1*0405, DQA1*0301, DQB1*0302) instead of HLA-DR15. ${ }^{5-7}$ The association between HLA-DR2 and MS has been observed mostly among northern European populations. ${ }^{8}$

A single vitamin D response element was identified in the HLA-DRB1 promoter region, advancing the idea of an interaction between vitamin D and HLA-DRB1. The role of this interaction in disease etiology has not been elucidated yet, but it can be hypothesized that a lack of vitamin $\mathrm{D}$ in early childhood can alter the expression levels of HLA-

Copyright (C) 2019 The Author(s). This is an open-access article distributed under the terms of the Creative Commons Attribution License (http:// creativecommons.org/licenses/by/4.0), which permits unrestricted use, distribution, and reproduction in any medium, provided the original work is properly cited. 
DRB1in the thymus, probably leading to a general reduction in the expression of disease-associated class II, such as HLA$\mathrm{DRB}^{*} 15: 01$, in the thymus during early life. This reduction might cause a loss of central tolerance, possibly increasing the risk of autoimmunity in later life. ${ }^{9}$ The lower frequency of the HLA-DRB1*15:01 allele in Asian and African populations, in comparison with Caucasian populations, may explain why a lower prevalence of MS is observed in races with dark skin living in temperate clines despite elevated vitamin D deficiency caused by the high ultraviolet-filtering capability of cutaneous melanin. ${ }^{9}$

HLA-DRB $1^{*} 1001^{10}$ and HLA-DRB ${ }^{*} 0101^{11}$ alleles have been reported to act as protective factors against MS in an Iranian and a Spanish population, respectively.

Variations in several miRNAs' SNPs appear to own genderspecific associations with MS susceptibility, course, and severity. Considering their post-transcriptional suppression of many genes' expression, these small single-stranded non-coding RNA molecules are evidenced to be of great importance in the regulation of several biological processes, including immune response, explaining the presence of dismantled miRNA expression as a common feature in several diseases involving the immune system. ${ }^{12}$ A study inspecting the expression levels of miRNAs involved in CD4+ T-cell activation detected the expression levels of miR-21, miR-146a, and miR-146b to be significantly higher in RRMS patients. ${ }^{13}$ Results from a study on 561 patients and 441 healthy controls suggest that miR-223 and miR-146A SNPs have correlations with MS susceptibility (the latter only in females). Moreover, miR-499A and miR-196A2 polymorphisms were observed to be significantly associated and not associated, in women and men, respectively, with a more severe course of the disease. ${ }^{14}$

\section{CYP24A1, CYP27B1, DBP, and VDR Genes}

CYP27B1 is the enzyme catalyzing the synthesis of $1,25(\mathrm{OH})_{2} \mathrm{D}_{3}$. CYP24A1 down-regulates $1,25(\mathrm{OH})_{2} \mathrm{D}_{3}$ levels through hydroxylation of both $1,25(\mathrm{OH})_{2} \mathrm{D}_{3}$ and $25(\mathrm{OH}) \mathrm{D}_{3}$ at C-24. Polymorphisms in CYP24A1, CYP27B1, vitamin D binding protein (DBP), and vitamin $\mathrm{D}$ receptor (VDR) genes may also appear effective in MS susceptibility, because they are involved in both functions and levels of vitamin D.

Variants in CYP27B1 appeared to be associated with higher MS risk, as heterozygosity for these mutations led to lower levels of calcitriol ${ }^{15}$; however, these results were later refuted since there were concerns as to the validity of the methods utilized in the study. Based on a meta-analysis, mostly among Caucasian populations, genetic polymorphism rs703842 in the CYP27B1 gene was shown to be significantly associated with MS risk, and the $\mathrm{C}$ allele was found to be correlated with reduced MS susceptibility. ${ }^{16}$ However, findings of a recent study on Sicilian patients diagnosed with MS do not provide evidence of an independent effect of vitamin D-related gene variants, DBP and CYP27B1, in the risk of MS. ${ }^{17}$ The C allele for rs-2248359 in the CYP24A1 gene is considered to be a risk factor as well. This allele is strongly associated with increased CYP24A1 expression in the frontal cortex of some MS patients, which results in lower levels of calcitriol in sites of CYP24A1 enzyme. ${ }^{18}$ The heterogeneity seen in the results of studies on the association between CYP24A1 and CYP27B1 polymorphisms and MS susceptibility may be explained by the different ethnic backgrounds of populations involved in the studies.

Other than transporting $25(\mathrm{OH}) \mathrm{D}_{3}$ to target sites, DBP is also capable of restricting the conversion of calcidiol to calcitriol by being tightly bound to calcidiol. Thus, factors affecting the functions or levels of DBP are estimated to influence the circulating $25(\mathrm{OH}) \mathrm{D}_{3}$ levels as well. However, its contribution to MS susceptibility is yet to be established, as studies inspecting this contribution have reported inconsistent results. The serum levels of DBP and calcitriol do not differ between patients and healthy controls, thus denying serum levels of bioavailable metabolite of vitamin $\mathrm{D}$ as a risk factor for MS. ${ }^{19}$ Another study, denied the association between DBP serum levels and MS susceptibility/activity, as no differences were observed in DBP concentrations among subjects (RRMS patients in relapse/remission phase) and healthy controls. ${ }^{20}$ Yet another study has reported DBP in MS patients to be of a higher serum level compared with healthy controls. ${ }^{21}$

The influence of the VDR gene polymorphisms on the risk of MS is not yet clear. The associations between BsmI, TaqI, ApaI, and FokI polymorphisms in the VDR gene and MS susceptibility have been investigated by many studies, often presenting controversial results. One recent study on 4013 MS patients found no association between these single nucleotide polymorphisms (SNPs) and MS risk in the general population; however, analysis of its subgroup suggested the A allele is associated with MS susceptibility in Asian populations. ${ }^{22}$ Another recent study involving 3593 patients reported TaqI polymorphism as a significant protective factor ${ }^{23}$. No relation between TaqI and FokI SNPs and increased MS risk was reported in a recent study by García-Martín et al. ${ }^{24}$ These SNPs were not found to be correlated with HLADRB1*15:01, either. ${ }^{24}$ A significant association was found between these two SNPs and MS risk in another recent study. ${ }^{25}$

\section{Environmental Factors}

Latitudinal and Seasonal Associations, UVB, and Vitamin D Despite the possible lack of accuracy in MS prevalence data, prior research has generally confirmed that MS is more prevalent in regions with higher latitude, which is associated with less sunlight intensity and lower UVB exposure in individuals. Individuals born in regions with (high/low) latitudes are reported to have a(n) (reduced/increased) MS risk after migrating to regions (closer to/further from) the equator in their second decade of life. ${ }^{26}$ Tao et al discovered an earlier age of MS onset among populations residing in regions with higher latitudes. ${ }^{27}$

A correlation has been reported between seasonal environmental factors and MS susceptibility. A study on 151978 MS patients born in the northern hemisphere purported that MS risk is increased strikingly among individuals born in April and reduced among those born in November and December, ${ }^{28}$ indicating the risk of MS is higher among those whose mothers experienced less sun exposure during pregnancy. Studies with fewer patients, however, do not necessarily support the previously reported association 
between season/month of birth and MS risk. ${ }^{29}$ Another study indicated an excess of births between May and October among MS patients. The peak of births of MS patients was shown to be in July and the nadir in December. ${ }^{30}$ Collectively, prenatal sun exposure may have an influence on the incidence of MS, most likely in combination with other environmental or genetic factors. ${ }^{31}$ Additionally, in the northern hemisphere, disease activity was reported to be augmented and reduced during summer/spring and winter/fall transitions among MS patients, respectively. ${ }^{32-35} \mathrm{~A}$ reverse correlation was reported in the southern hemisphere, ${ }^{36,37}$ as the meteorological seasons in the southern hemisphere are also opposite to those in the northern hemisphere. Vitamin D is suggested as a contributor to some, but not all, geographical and seasonal associations observed in MS. ${ }^{38}$ The Epstein-Barr virus (EBV) seropositivity is evidenced to own a positive association with latitude in an MS-independent manner. ${ }^{39,40}$

Lower UVB exposure is considered associated with higher MS susceptibility and is obviously associated with lower annual mean UVB irradiation and average winter UVB. ${ }^{41}$ Given the information on immunomodulatory effects of vitamin $\mathrm{D}$ and its protective role against autoimmune diseases, it is possible that lower UVB exposure could increase the risk of MS by causing less cholecalciferol production in the skin. Another possible explanation illustrates the independent role of UVB on immunomodulation, as it may stimulate dendritic cells present in the skin, producing interleukin-10 (IL-10; an anti-inflammatory cytokine considered protective against the disease). IL-10 can stimulate local regulatory T-cells (Tregs) present in the skin and Tregs in the lymph nodes. These activated Tregs can exert their immunomodulatory functions within CNS after entering the bloodstream. ${ }^{42}$

The perception of vitamin $\mathrm{D}$ insufficiency (or deficiency) as a major MS risk factor is attributable to its interaction with some other risk factors, such as HLA haplotypes which are related to higher MS risk. Furthermore, non-classical functions of vitamin $\mathrm{D}$, such as the regulation of both innate and adaptive immunity, the presence and possible neuroprotective actions in CNS cells, and its interaction with other risk factors have contributed to the perception mentioned above.

EBV, Human Endogenous Retrovirus and Human Herpes Virus Type 6 (HHV-6)

EBV (HHV-4) infection has been shown to be significantly associated with MS. ${ }^{43,44}$ It has been suggested that EBV seropositivity and elevation of EBV-specific antibody titers precede the onset of the disease by at least a few years. ${ }^{45}$ Increased anti-EBNA1 serum levels were perceived to be significantly associated with a number of risk alleles, including rs2744148, rs11154801, rs1843938, and rs7200786. ${ }^{46}$ This infectious agent has shown consistency regarding its association with MS development more than any other microbe. ${ }^{47}$ Considering this consistency, EBV vaccination and antiviral drugs utilization in treatment procedures appear necessary for the prevention and inhibition of disease progression, respectively. ${ }^{48}$ It is mostly asymptomatic during childhood, but commonly symptomatic and causing infectious mononucleosis (IM) during adolescence and adulthood. ${ }^{49}$ After a late EBV infection, high serum levels of EBV-specific antibodies and a history of IM appear to be significantly associated with increased risk for MS. ${ }^{50}$ In a recent study, the incidence of IM and genetic susceptibility of high titers of EBNA-1 IgG manifested a positive time correlation with MS development in female patients with post-pubertal infection and male patients and a negative time correlation among female patients with pre-pubescent infection. ${ }^{51}$ Certain variants in MS-associated polymorphisms, including GC and CC genotypes in miR-146a (rs2910164), are suggested to amplify this correlation and to predict the conversion to MS and also relapse rate. ${ }^{52} \mathrm{EBV}$ exerts its pathogenic effects most likely by infecting B-cells and not by molecular mimicry; treatment with B-cell-depleting drugs, such as rituximab and ocrelizumab, which eradicate EBV-infected B-cells has been shown to reduce disease activity markers. ${ }^{53,54}$ B-cells present in cerebrospinal fluid (CSF) are reported to be infected with EBV in MS patients..$^{55}$ EBV-infected B lymphocytes tend to have higher antigen presenting capacity, co-stimulate T-cells, attract inflammatory cells, and alter the anti-inflammatory/pro-inflammatory balance, which leads to lower tolerogenic responses like IL-10 production by regulatory B-cells (Bregs). ${ }^{55,56}$ Consumption of high-dose vitamin $\mathrm{D}$ supplements seems to reduce the anti-EBNA1 serum levels in RRMS patients..$^{57,58}$

Infections with human endogenous retroviruses (HERVs) and HHV-6 are suggested to precede the disease onset and/ or increase the disability progression rate. ${ }^{59}$ Two antigenic peptides present on the HERV-W envelope surface have been reported to be highly recognized by antibodies in MS patients in comparison with healthy subjects. ${ }^{60} \mathrm{~A}$ recent experiment suggested a molecular mimicry in epitopes from envelope proteins of the HERV-W family and myelin proteins, confirmation of which requires further experiments to test the ability of these epitopes to activate helper T-cells (Th-cells). ${ }^{61}$

A systematic review and meta-analysis on 39 studies demonstrated a significant association between MS and infection with HHV-6. ${ }^{43}$ The presence of anti-HHV-6 IgG in the CSF of MS patients, especially those with the progressive form of the disease, has been confirmed. ${ }^{62}$ Higher anti-HHV-6 IgG titers in MS patients have been reported to be associated with higher relapse risk. ${ }^{63}$

\section{Cigarette Smoking}

Apart from the suggested positive correlation of MS risk with cigarette smoking, nicotine consumption may reduce this risk if not smoked. ${ }^{64,65}$ Some known effects of other substances found in cigarette smoke encompass direct tissuedamage, increased apoptosis, and pro-inflammatory actions. ${ }^{5}$ The association between smoking and MS susceptibility was investigated using Bradford Hill's criteria for causation in a recent study conducted by Degelman and Herman, in which an increased risk for MS was observed in smokers. ${ }^{66}$ Another recent study provided information regarding the effects of different smoking habits on MS risk, indicating a dose-response relationship between these two variables. ${ }^{67}$ 
It was deduced by one study that the increased MS risk associated with smoking remains up to 5 years after cessation of smoking. ${ }^{68}$ The effect of maternal smoking on MS risk is not clarified yet, but one case control study showed a higher risk, ${ }^{69}$ and a second showed no association. ${ }^{70}$

The association between increased MS risk and higher antiEBNA titers is augmented in ever smokers compared with never smokers. ${ }^{71}$ The interaction between MS susceptibility alleles and cigarette smoke exposure is still a matter of debate, as one study suggests there is no such interaction, ${ }^{72}$ and another study has found these interactions possible. ${ }^{73}$ The analysis of 6 independent studies in 5 countries indicated that smokers lacking HLA-A*02 and carrying HLA-DRB1* 15 had a 13-fold greater risk of developing MS than never smokers who lack HLA-DRB ${ }^{*} 15$ and carry HLA-A*02. ${ }^{74}$

Cigarette smoking has been associated with disease activity in RRMS patients. In a recent study, it was noted that the progression of the expanded disability scale score was faster in daily smokers than in non-smokers. ${ }^{75}$ This effect on disease progression is assumed to be caused by a reduction in $\mathrm{CD}^{+}, \mathrm{CD} 25^{+}$, and other regulatory cells. This reduction is explainable by two pathways suggested in a recent study: I) increased production of IL-6 and IL-13, attributable to the decreased activity of indoleamine 2,3-dioxygenase, and II) Elevation in the expression and activity of the renin angiotensin system, inducing an increase in IL-17 and IL-22, resulting in the higher production of chemokines in smoking patients. $^{75}$

The association between cigarette smoking and disease progression in PPMS is not clarified yet. A recent study showed no effect exerted by smoking on disability accumulation in PPMS, suggesting that the disability progression in PPMS involves different underlying pathomechanisms from that in RRMS ${ }^{76}$ A clinical cohort found a significantly higher risk of SPMS onset in ever smokers, occurring approximately 4 years earlier. ${ }^{77}$

\section{Obesity}

Studies have demonstrated a significant association between obesity in adolescence and increased MS risk. ${ }^{78-83} \mathrm{~A}$ two-fold increase of MS risk in obese individuals (BMI $\geq 30 \mathrm{~kg} / \mathrm{m}^{2}$ ) compared with those with normal BMIs has consistently been observed. ${ }^{83}$

Obesity has been shown to be associated with lower $25(\mathrm{OH}) \mathrm{D}$ serum levels in individuals, irrespective of latitude, age, etc. ${ }^{84} \mathrm{~A}$ recent meta-analysis suggested lower serum levels of 25(OH)D and higher BMI to be significantly and independently correlated with higher susceptibility to pediatric-onset MS.$^{85}$ Female individuals with a higher BMI during childhood and adolescence tend to experience an earlier menarche, ${ }^{86-89}$ which is suggested to be associated with an earlier MS-onset in females. ${ }^{90}$ Whether early puberty in females is a contributing factor to MS development or an incident involved in the pathomechanisms of MS is yet to be illuminated.

Although childhood obesity has not been established as a factor favoring the disease onset, considering that approximately $66 \%$ of children placed among the highest
BMI quartile do not descend to lower quartiles during youngadulthood, ${ }^{91}$ an estimation by Ascherio and Munger suggests a $15 \%$ reduction in MS cases following the elimination of childhood obesity, ${ }^{92}$ concluded from the two-fold increased MS risk in individuals with childhood obesity and a $17 \%$ spread of obesity among adolescents. ${ }^{93}$

Adipokines such as leptin, resistin, and visfatin have been suggested to be the links between metabolic status and the immune system in individuals, mediating the immune response towards a pro-inflammatory profile when increased in serum levels, consequently augmenting disease severity in RRMS patients. ${ }^{94}$ Adiponectin was observed to be of lower serum levels in RRMS patients compared to healthy controls. ${ }^{95}$

\section{Human Cytomegalovirus (HCMV) and Helicobacter pylori}

Several studies have attempted to determine the association between HCMV (HHV-5) infection and disease activity in MS patients. However, the results regarding this association remain inconsistent. It was suggested by a recent study that HCMV possibly intensifies symptoms in MS patients. ${ }^{96}$ Two recent studies involving Iranian populations have also suggested a key role for HCMV in the pathogenesis of MS, ${ }^{97,98}$ while another study suggested a possible beneficial role for HCMV in attenuating the risk of disability progression in MS patients, showing virus-driven NKG2C+ natural killer cell expansion. ${ }^{99}$

Infection with $\mathrm{H}$. pylori and MS susceptibility have been suggested by some studies to be negatively correlated, but there is no consensus over this possible correlation. Observing a significantly lower prevalence of $\mathrm{H}$. pylori infection among MS patients, a recent study involving 1902 MS patients concluded that infection with $\mathrm{H}$. pylori is likely to be a protective factor against developing MS. ${ }^{100}$ Another recent study with 2806 participants illustrated the same negative correlation between $H$. pylori infection and MS susceptibility, especially in western populations, ${ }^{101}$ possibly through the induction of Tregs and Th2 lymphocytes by $\mathrm{H}$. pylori as an evasion mechanism and attenuation of Th1/ Th17-mediated pro-inflammatory responses by Tregs. A cascade reaction involving malabsorption of vitamin $B_{12}$ and folic acid, reactive oxygen species induction, and elevation of plasma homocysteine following the propagation of $\mathrm{H}$. pylori can increase the risk of Alzheimer's disease (AD) by damaging the brain-blood barrier, which can result in the accumulation of amyloid- $\beta$ in the brain. Therefore, $H$. pylori remains a pathogenic agent in neurological disorders such as $\mathrm{AD}$, despite its potential negative association with MS. ${ }^{102}$

\section{Alcohol, Caffeine, and Nicotine}

In case of MS susceptibility, studies have manifested no association between alcohol consumption and increased MS risk. ${ }^{103,104}$ Regarding the correlation of disease severity in RRMS patients with alcohol consumption, one recent study suggested that the cessation of alcohol consumption could potentially be beneficial, ${ }^{75}$ and the results yielded an inverse association between drinking alcoholic beverages and the progression rate among patients diagnosed with SPMS. In PPMS patients, however, there was no association observed, ${ }^{105}$ 
supporting the idea that different mechanisms are involved in the disability progression of PPMS and SPMS patients. A more recent study found total alcohol and red wine consumption to be negatively associated with cross-sectional neurologic disability progression. ${ }^{106}$

The consumption of coffee is suggested to have an inverse association with disease progression rate in SPMS, but not in PPMS. ${ }^{105}$ Two independent studies yielded similar results regarding the effect of coffee consumption on MS susceptibility, which they reported to be protective. ${ }^{107}$ It is possible that the mechanisms underlying this effect involve the neuroprotective and anti-inflammatory functions of caffeine (the major component of coffee) in neurodegenerative diseases. ${ }^{108}$

Given its neuroprotective properties in CNS, ${ }^{109}$ nicotine consumption is suggested to own therapeutic roles in the management of Parkinson's disease and to be associated with a lower risk of developing the disease. ${ }^{110,111}$ Other than its involvement in the pathogenesis and treatment of Parkinson's disease, it has been suggested that the nicotinic acetylcholine receptor is involved in the pathomechanisms through which AD progresses. ${ }^{12}$ Some studies have tested this role in rat models of MS (experimental autoimmune encephalomyelitis) and have determined that nicotine is a protective agent in both MS susceptibility and severity. ${ }^{113}$ Two case control studies (7883 cases, 9437 controls) executed in Sweden, in which the use of moist snuff (a smokeless tobacco product placed between the lips and gums, resulting in high blood concentrations of nicotine) is common, investigated the MS risk among populations with different snuff consumption habits and provided evidence for the hypothesized involvement of the anti-inflammatory and immunomodulatory effects of nicotine, as it was noticed to reduce the risk of developing MS in both never-smokers and smokers. ${ }^{69}$ A recent study suggested the combination of mesenchymal stem cells (MSCs) and nicotine for the further improvement of MS therapeutics as the increased IL-10 production and significantly reduced production of pro-inflammatory cytokines, such as IL-17 and tumor necrosis factor (TNF)- $\alpha$, were observed in rats with EAE treated with this combined therapy. ${ }^{114}$

\section{Conclusions}

We reviewed recent studies investigating factors, the involvement of which has been proposed in numerous research papers. Our efforts toward achieving a relatively complete understanding of these suggested factors will guide us through the process of designing social and global programs, aiming to minimize the exposure of those recognized as "susceptible" to the environmental conditions we assume to be threatening. Studying the underlying genetic information on patients, the person-to-person approach further enables clinicians around the world to monitor the interactions between the risk factors encoded in individuals' genomes with the interventions they are receiving as well as the environment in which they are spending most of their daily time. For instance, health promotion endeavors are essential for encouraging the populations considered in danger (e.g., smokers) to discontinue the behaviors or presence in risky environments. For viral infections suggested to be involved in the disease pathomechanisms, further research is needed to elucidate their roles to a measure of precision that will allow the execution of actions such as vaccination with a lucid vision of the balance of benefits and risks in such undertakings.

\section{Authors' Contributions}

All authors contributed equally to this study.

\section{Conflict of Interest Disclosures}

All authors declare that they have no conflicts of interest.

\section{References}

1. Thompson AJ, Banwell BL, Barkhof F, et al. Diagnosis of multiple sclerosis: 2017 revisions of the McDonald criteria. Lancet Neurol. 2018;17(2):162-173. doi:10.1016/S1474-4422(17)30470-2.

2. Goldenberg MM. Multiple sclerosis review. P T. 2012;37(3):175184.

3. Trapp BD, Vignos M, Dudman J, et al. Cortical neuronal densities and cerebral white matter demyelination in multiple sclerosis: a retrospective study. Lancet Neurol. 2018;17(10):870-884. doi:10.1016/S1474-4422(18)30245-X.

4. Didonna A, Oksenberg JR. Genetic determinants of risk and progression in multiple sclerosis. Clin Chim Acta. 2015;449:1622. doi:10.1016/j.cca.2015.01.034.

5. O'Gorman C, Lucas R, Taylor B. Environmental risk factors for multiple sclerosis: a review with a focus on molecular mechanisms. Int J Mol Sci. 2012;13(9):11718-11752. doi:10.3390/ ijms130911718.

6. Marrosu MG, Murru MR, Costa G, Murru R, Muntoni F, Cucca F. DRB1-DQA1-DQB1 loci and multiple sclerosis predisposition in the Sardinian population. Hum Mol Genet. 1998;7(8):1235-1237. doi:10.1093/hmg/7.8.1235.

7. Haegert DG, Muntoni F, Murru MR, Costa G, Francis GS, Marrosu MG. HLA-DQA1 and -DQB1 associations with multiple sclerosis in Sardinia and French Canada: evidence for immunogenetically distinct patient groups. Neurology. 1993;43(3 Pt 1):548-552. doi:10.1212/wnl.43.3_part_1.548.

8. Robertson N, Compston A. Surveying multiple sclerosis in the United Kingdom. J Neurol Neurosurg Psychiatry. 1995;58(1):2-6. doi:10.1136/jnnp.58.1.2.

9. Handunnetthi L, Ramagopalan SV, Ebers GC. Multiple sclerosis, vitamin D, and HLA-DRB1*15. Neurology. 2010;74(23):19051910. doi:10.1212/WNL.0b013e3181e24124.

10. Kollaee A, Ghaffarpor M, Ghlichnia HA, Ghaffari SH, Zamani $M$. The influence of the HLA-DRB1 and HLA-DQB1 allele heterogeneity on disease risk and severity in Iranian patients with multiple sclerosis. Int J Immunogenet. 2012;39(5):414-422. doi:10.1111/j.1744-313X.2012.01104.x.

11. Fernandez O, R-Antiguedad A, Pinto-Medel MJ, et al. HLA class II alleles in patients with multiple sclerosis in the Biscay province (Basque Country, Spain). J Neurol. 2009;256(12):1977-1988. doi:10.1007/s00415-009-5223-2.

12. Gracias DT, Katsikis PD. MicroRNAs: key components of immune regulation. Adv Exp Med Biol. 2011;780:15-26. doi:10.1007/9781-4419-5632-3_2.

13. Fenoglio C, Cantoni C, De Riz M, et al. Expression and genetic analysis of miRNAs involved in CD4+ cell activation in patients with multiple sclerosis. Neurosci Lett. 2011;504(1):9-12. doi:10.1016/j.neulet.2011.08.021.

14. Kiselev I, Bashinskaya V, Kulakova O, et al. Variants of MicroRNA genes: gender-specific associations with multiple sclerosis risk and severity. Int J Mol Sci. 2015;16(8):20067-20081. doi:10.3390/ ijms160820067.

15. Ramagopalan SV, Dyment DA, Cader MZ, et al. Rare variants in the CYP27B1 gene are associated with multiple sclerosis. Ann 
Neurol. 2011;70(6):881-886. doi:10.1002/ana.22678.

16. Jiang $\mathrm{T}$, Li L, Wang $\mathrm{Y}$, et al. The association between genetic polymorphism rs703842 in CYP27B1 and multiple sclerosis: a meta-analysis. Medicine (Baltimore). 2016;95(19):e3612. doi:10.1097/md.0000000000003612.

17. Agnello L, Scazzone C, Lo Sasso B, et al. VDBP, CYP27B1, and 25-hydroxyvitamin $\mathrm{D}$ gene polymorphism analyses in a group of Sicilian multiple sclerosis patients. Biochem Genet. 2017;55(2):183-192. doi:10.1007/s10528-016-9783-4.

18. Ramasamy A, Trabzuni D, Forabosco P, et al. Genetic evidence for a pathogenic role for the vitamin D3 metabolizing enzyme CYP24A1 in multiple sclerosis. Mult Scler Relat Disord. 2014;3(2):211-219. doi:10.1016/j.msard.2013.08.009.

19. Behrens JR, Rasche L, Giess RM, et al. Low 25-hydroxyvitamin D, but not the bioavailable fraction of 25-hydroxyvitamin D, is a risk factor for multiple sclerosis. Eur J Neurol. 2016;23(1):62-67. doi:10.1111/ene.12788.

20. Smolders J, Peelen E, Thewissen M, Menheere P, Damoiseaux J, Hupperts R. Circulating vitamin D binding protein levels are not associated with relapses or with vitamin D status in multiple sclerosis. Mult Scler. 2014;20(4):433-437. doi:10.1177/1352458513500552.

21. Rinaldi AO, Sanseverino I, Purificato C, et al. Increased circulating levels of vitamin D binding protein in MS patients. Toxins (Basel). 2015;7(1):129-137. doi:10.3390/toxins7010129.

22. Zhang YJ, Zhang L, Chen SY, et al. Association between VDR polymorphisms and multiple sclerosis: systematic review and updated meta-analysis of case-control studies. Neurol Sci. 2018;39(2):225-234. doi:10.1007/s10072-017-3175-3.

23. Chen $\mathrm{XL}$, Zhang $\mathrm{ML}$, Zhu $\mathrm{L}$, et al. Vitamin $\mathrm{D}$ receptor gene polymorphisms and the risk of multiple sclerosis: An updated meta-analysis. Microb Pathog. 2017;110:594-602. doi:10.1016/j. micpath.2017.08.002.

24. García-Martín E, Agundez JA, Martinez C, et al. Vitamin D3 receptor ( VDR) gene rs2228570 (Fok1) and rs731236 (Taq1) variants are not associated with the risk for multiple sclerosis: results of a new study and a meta-analysis. PLoS One. 2013;8(6):e65487. doi:10.1371/journal.pone.0065487.

25. Abdollahzadeh R, Moradi Pordanjani P, Rahmani F, Mashayekhi F, Azarnezhad A, Mansoori Y. Association of VDR gene polymorphisms with risk of relapsing-remitting multiple sclerosis in an Iranian Kurdish population. Int J Neurosci. 2018;128(6):505511. doi:10.1080/00207454.2017.1398158.

26. Handel AE, Giovannoni G, Ebers GC, Ramagopalan SV. Environmental factors and their timing in adult-onset multiple sclerosis. Nat Rev Neurol. 2010;6(3):156-166. doi:10.1038/ nrneurol.2010.1.

27. Tao C, Simpson S Jr, van der Mei I, et al. Higher latitude is significantly associated with an earlier age of disease onset in multiple sclerosis. J Neurol Neurosurg Psychiatry. 2016;87(12):1343-1349. doi:10.1136/jnnp-2016-314013.

28. Dobson R, Giovannoni G, Ramagopalan S. The month of birth effect in multiple sclerosis: systematic review, meta-analysis and effect of latitude. J Neurol Neurosurg Psychiatry. 2013;84(4):427432. doi:10.1136/jnnp-2012-303934.

29. Eliasdottir O, Hildeman A, Longfils M, Nerman O, Lycke J. A nationwide survey of the influence of month of birth on the risk of developing multiple sclerosis in Sweden and Iceland. J Neurol. 2018;265(1):108-114. doi:10.1007/s00415-017-8665-y.

30. Sidhom Y, Kacem I, Bayoudh L, et al. Season of birth and multiple sclerosis in Tunisia. Mult Scler Relat Disord. 2015;4(6):491-494. doi:10.1016/j.msard.2015.08.002.

31. Villar-Quiles RN, Matias-Guiu JA, Ortega G, Gonzalez-Suarez I, Oreja-Guevara C, Matias-Guiu J. Analysis of the relationship between the month of birth and risk of multiple sclerosis in a Spanish population. Eur Neurol. 2016;76(5-6):202-209. doi:10.1159/000449246.

32. Auer DP, Schumann EM, Kumpfel T, Gossl C, Trenkwalder C. Seasonal fluctuations of gadolinium-enhancing magnetic resonance imaging lesions in multiple sclerosis. Ann Neurol. 2000;47(2):276277 . ana28>3.0.co;2-1.

33. Handel $A E$, Disanto $G$, Jarvis $L$, et al. Seasonality of admissions with multiple sclerosis in Scotland. Eur J Neurol. 2011;18(8):11091111. doi:10.1111/j.1468-1331.2010.03318.x.

34. Jin $Y$, de Pedro-Cuesta J, Soderstrom M, Stawiarz L, Link H. Seasonal patterns in optic neuritis and multiple sclerosis: a metaanalysis. J Neurol Sci. 2000;181(1-2):56-64. doi:10.1016/S0022510X(00)00408-1.

35. Meier DS, Balashov KE, Healy B, Weiner HL, Guttmann CR. Seasonal prevalence of MS disease activity. Neurology. 2010;75(9):799-806. doi:10.1212/WNL.0b013e3181f0734c.

36. Damasceno A, Von Glehn F, de Deus-Silva L, Damasceno BP. Monthly variation of multiple sclerosis activity in the southern hemisphere: analysis from 996 relapses in Brazil. Eur J Neurol. 2012;19(4):660-662. doi:10.1111/j.1468-1331.2011.03543.x.

37. Tremlett $H$, van der Mei IA, Pittas F, et al. Monthly ambient sunlight, infections and relapse rates in multiple sclerosis. Neuroepidemiology. 2008;31(4):271-279. doi:10.1159/000166602.

38. Dorr J, Doring A, Paul F. Can we prevent or treat multiple sclerosis by individualised vitamin D supply? Epma j. 2013;4(1):4 doi:10.1186/1878-5085-4-4.

39. Disanto G, Pakpoor J, Morahan JM, et al. Epstein-Barr virus, latitude and multiple sclerosis. Mult Scler. 2013;19(3):362-365. doi:10.1177/1352458512451942.

40. Lossius A, Riise T, Pugliatti $M$, et al. Season of infectious mononucleosis and risk of multiple sclerosis at different latitudes; the EnvIMS Study. Mult Scler. 2014;20(6):669-674. doi:10.1177/1352458513505693.

41. Orton SM, Wald L, Confavreux C, et al. Association of UV radiation with multiple sclerosis prevalence and sex ratio in France. Neurology. 2011;76(5):425-431. doi:10.1212/ WNL.0b013e31820a0a9f.

42. DeLuca HF, Plum L. UVB radiation, vitamin D and multiple sclerosis. Photochem Photobiol Sci. 2017;16(3):411-415. doi:10.1039/c6pp00308g.

43. Pormohammad A, Azimi T, Falah F, Faghihloo E. Relationship of human herpes virus 6 and multiple sclerosis: A systematic review and meta-analysis. J Cell Physiol. 2018;233(4):2850-2862. doi:10.1002/jcp.26000.

44. Handel AE, Williamson AJ, Disanto G, Handunnetthi L, Giovannoni G, Ramagopalan SV. An updated meta-analysis of risk of multiple sclerosis following infectious mononucleosis. PLoS One. 2010;5(9). doi:10.1371/journal.pone.0012496.

45. Levin LI, Munger KL, Rubertone MV, et al. Temporal relationship between elevation of epstein-barr virus antibody titers and initial onset of neurological symptoms in multiple sclerosis. Jama. 2005;293(20):2496-2500. doi:10.1001/jama.293.20.2496.

46. Kreft KL, Van Nierop GP, Scherbeijn SMJ, Janssen M, Verjans G, Hintzen RQ. Elevated EBNA-1 IgG in MS is associated with genetic MS risk variants. Neurol Neuroimmunol Neuroinflamm. 2017;4(6):e406. doi:10.1212/nxi.0000000000000406.

47. Ascherio A, Munger KL. Environmental risk factors for multiple sclerosis. Part I: the role of infection. Ann Neurol. 2007;61(4):288299. doi:10.1002/ana.21117.

48. Pender MP. Preventing and curing multiple sclerosis by controlling Epstein-Barr virus infection. Autoimmun Rev. 2009;8(7):563-568. doi:10.1016/j.autrev.2009.01.017.

49. Fernandez-Menendez S, Fernandez-Moran M, Fernandez-Vega I, Perez-Alvarez A, Villafani-Echazu J. Epstein-Barr virus and multiple sclerosis. From evidence to therapeutic strategies. J Neurol Sci. 2016;361:213-219. doi:10.1016/j.jns.2016.01.013.

50. Haahr S, Plesner AM, Vestergaard BF, Hollsberg P. A role of late Epstein-Barr virus infection in multiple sclerosis. Acta Neurol Scand. 2004;109(4):270-275. doi:10.1046/j.16000404.2003.00221.x.

51. Endriz J, Ho PP, Steinman L. Time correlation between 
mononucleosis and initial symptoms of MS. Neurol Neuroimmunol Neuroinflamm. 2017;4(3):e308. doi:10.1212/ nxi.0000000000000308.

52. Zhou Y, Chen M, Simpson S Jr, et al. Common genetic variation within miR-146a predicts disease onset and relapse in multiple sclerosis. Neurol Sci. 2018;39(2):297-304. doi:10.1007/s10072017-3177-1.

53. Hauser SL, Waubant E, Arnold DL, et al. B-cell depletion with rituximab in relapsing-remitting multiple sclerosis. N Engl J Med. 2008;358(7):676-688. doi:10.1056/NEJMoa0706383.

54. Kappos L, Li D, Calabresi PA, et al. Ocrelizumab in relapsingremitting multiple sclerosis: a phase 2, randomised, placebocontrolled, multicentre trial. Lancet. 2011;378(9805):1779-1787. doi:10.1016/s0140-6736(11)61649-8.

55. Serafini B, Rosicarelli B, Franciotta D, et al. Dysregulated EpsteinBarr virus infection in the multiple sclerosis brain. J Exp Med. 2007;204(12):2899-2912. doi:10.1084/jem.20071030.

56. Muris AH, Smolders J, Rolf L, Thewissen M, Hupperts R, Damoiseaux J. Immune regulatory effects of high dose vitamin D3 supplementation in a randomized controlled trial in relapsing remitting multiple sclerosis patients receiving IFNbeta; the SOLARIUM study. J Neuroimmunol. 2016;300:47-56. doi:10.1016/j.jneuroim.2016.09.018.

57. Rosjo E, Lossius A, Abdelmagid N, et al. Effect of high-dose vitamin D3 supplementation on antibody responses against Epstein-Barr virus in relapsing-remitting multiple sclerosis. Mult Scler. 2017;23(3):395-402. doi:10.1177/1352458516654310.

58. Rolf L, Muris AH, Mathias A, et al. Exploring the effect of vitamin D3 supplementation on the anti-EBV antibody response in relapsingremitting multiple sclerosis. Mult Scler. 2018;24(10):1280-1287. doi:10.1177/1352458517722646.

59. de la Hera B, Varade J, Garcia-Montojo M, et al. Human endogenous retrovirus HERV-Fc1 association with multiple sclerosis susceptibility: a meta-analysis. PLoS One. 2014;9(3):e90182. doi:10.1371/journal.pone.0090182.

60. Mameli G, Cossu D, Cocco E, et al. Epitopes of HERV-Wenv induce antigen-specific humoral immunity in multiple sclerosis patients. J Neuroimmunol. 2015;280:66-68. doi:10.1016/j. jneuroim.2015.03.003.

61. Ramasamy R, Joseph B, Whittall T. Potential molecular mimicry between the human endogenous retrovirus $W$ family envelope proteins and myelin proteins in multiple sclerosis. Immunol Lett. 2017;183:79-85. doi:10.1016/j.imlet.2017.02.003.

62. Alenda R, Alvarez-Lafuente R, Costa-Frossard L, et al. Identification of the major HHV-6 antigen recognized by cerebrospinal fluid IgG in multiple sclerosis. Eur J Neurol. 2014;21(8):1096-1101. doi:10.1111/ene.12435.

63. Simpson S Jr, Taylor B, Dwyer DE, et al. Anti-HHV-6 IgG titer significantly predicts subsequent relapse risk in multiple sclerosis. MultScler.2012;18(6):799-806. doi:10.1177/1352458511428081.

64. Hedstrom AK, Hillert J, Olsson T, Alfredsson L. Nicotine might have a protective effect in the etiology of multiple sclerosis. Mult Scler. 2013;19(8):1009-1013. doi:10.1177/1352458512471879.

65. Nizri E, Irony-Tur-Sinai M, Lory O, Orr-Urtreger A, Lavi E, Brenner T. Activation of the cholinergic anti-inflammatory system by nicotine attenuates neuroinflammation via suppression of Th1 and Th17 responses. J Immunol. 2009;183(10):6681-6688. doi:10.4049/ jimmunol.0902212.

66. Degelman ML, Herman KM. Smoking and multiple sclerosis: A systematic review and meta-analysis using the Bradford Hill criteria for causation. Mult Scler Relat Disord. 2017;17:207-216. doi:10.1016/j.msard.2017.07.020.

67. Poorolajal J, Bahrami M, Karami M, Hooshmand E. Effect of smoking on multiple sclerosis: a meta-analysis. J Public Health (Oxf). 2017;39(2):312-320. doi:10.1093/pubmed/fdw030.

68. Hedstrom AK, Baarnhielm M, Olsson T, Alfredsson L. Tobacco smoking, but not Swedish snuff use, increases the risk of multiple sclerosis. Neurology. 2009;73(9):696-701. doi:10.1212/ WNL.0b013e3181b59c40.
69. Mueller BA, Nelson JL, Newcomb PA. Intrauterine environment and multiple sclerosis: a population- based case-control study. Mult Scler. 2013;19(1):106-111. doi:10.1177/1352458512447869.

70. Montgomery SM, Bahmanyar S, Hillert J, Ekbom A, Olsson T. Maternal smoking during pregnancy and multiple sclerosis amongst offspring. Eur J Neurol. 2008;15(12):1395-1399. doi:10.1111/j.1468-1331.2008.02331.x.

71. Simon KC, van der Mei IA, Munger KL, et al. Combined effects of smoking, anti-EBNA antibodies, and HLA-DRB1*1501 on multiple sclerosis risk. Neurology. 2010;74(17):1365-1371. doi:10.1212/ WNL.0b013e3181dad57e.

72. Jafari N, Hoppenbrouwers IA, Hop WC, Breteler MM, Hintzen RQ. Cigarette smoking and risk of MS in multiplex families. Mult Scler. 2009;15(11):1363-1367. doi:10.1177/1352458509345907.

73. Hedstrom AK, Sundqvist E, Baarnhielm M, et al. Smoking and two human leukocyte antigen genes interact to increase the risk for multiple sclerosis. Brain. 2011;134(Pt 3):653-664. doi:10.1093/ brain/awq371.

74. Hedstrom AK, Katsoulis M, Hossjer $\mathrm{O}$, et al. The interaction between smoking and HLA genes in multiple sclerosis: replication and refinement. Eur J Epidemiol. 2017;32(10):909-919. doi:10.1007/s10654-017-0250-2.

75. Paz-Ballesteros WC, Monterrubio-Flores EA, de Jesus Flores-Rivera J, Corona-Vazquez T, Hernandez-Giron C. Cigarette Smoking, Alcohol Consumption and Overweight in Multiple Sclerosis: Disability Progression. Arch Med Res. 2017;48(1):113-120. doi:10.1016/j.arcmed.2017.03.002.

76. Javizian O, Metz LM, Deighton S, Koch MW. Smoking does not influence disability accumulation in primary progressive multiple sclerosis. Eur J Neurol. 2017;24(4):624-630. doi:10.1111/ ene.13262.

77. O'Gorman CM, Broadley SA. Smoking increases the risk of progression in multiple sclerosis: A cohort study in Queensland, Australia. J Neurol Sci. 2016;370:219-223. doi:10.1016/j. jns.2016.09.057.

78. Gianfrancesco MA, Barcellos LF. Obesity and multiple sclerosis susceptibility: a review. J Neurol Neuromedicine. 2016;1(7):1-5. doi:10.29245/2572.942X/2016/7.1064.

79. Gianfrancesco MA, Acuna B, Shen L, et al. Obesity during childhood and adolescence increases susceptibility to multiple sclerosis after accounting for established genetic and environmental risk factors. Obes Res Clin Pract. 2014;8(5):e435447. doi:10.1016/j.orcp.2014.01.002.

80. Hedstrom AK, Lima Bomfim I, Barcellos L, et al. Interaction between adolescent obesity and HLA risk genes in the etiology of multiple sclerosis. Neurology. 2014;82(10):865-872. doi:10.1212/ wnl.0000000000000203.

81. Hedstrom AK, Olsson T, Alfredsson L. High body mass index before age 20 is associated with increased risk for multiple sclerosis in both men and women. Mult Scler. 2012;18(9):13341336. doi:10.1177/1352458512436596.

82. Munger KL, Bentzen J, Laursen B, et al. Childhood body mass index and multiple sclerosis risk: a long-term cohort study. Mult Scler. 2013;19(10):1323-1329. doi:10.1177/1352458513483889.

83. Munger KL, Chitnis T, Ascherio A. Body size and risk of MS in two cohorts of US women. Neurology. 2009;73(19):1543-1550. doi:10.1212/WNL.0b013e3181c0d6e0.

84. Pereira-Santos M, Costa PR, Assis AM, Santos CA, Santos DB. Obesity and vitamin $D$ deficiency: a systematic review and metaanalysis. Obes Rev. 2015;16(4):341-349. doi:10.1111/obr.12239.

85. Gianfrancesco MA, Stridh P, Rhead B, et al. Evidence for a causal relationship between low vitamin $\mathrm{D}$, high $\mathrm{BMI}$, and pediatriconset MS. Neurology. 2017;88(17):1623-1629. doi:10.1212/ wnl.0000000000003849.

86. Cheung CC, Thornton JE, Kuijper JL, Weigle DS, Clifton DK, Steiner RA. Leptin is a metabolic gate for the onset of puberty in the female rat. Endocrinology. 1997;138(2):855-858. doi:10.1210/ endo.138.2.5054.

87. Lee JM, Appugliese D, Kaciroti N, Corwyn RF, Bradley RH, 
Lumeng JC. Weight status in young girls and the onset of puberty. Pediatrics. 2007;119(3):e624-630. doi:10.1542/peds.2006-2188.

88. Matkovic V, Ilich JZ, Skugor M, et al. Leptin is inversely related to age at menarche in human females. J Clin Endocrinol Metab. 1997;82(10):3239-3245. doi:10.1210/jcem.82.10.4280.

89. Chitnis T. Role of puberty in multiple sclerosis risk and course. Clin Immunol.2013;149(2):192-200.doi:10.1016/j.clim.2013.03.014.

90. Ramagopalan SV, Valdar W, Criscuoli M, et al. Age of puberty and the risk of multiple sclerosis: a population based study. Eur J Neurol. 2009;16(3):342-347.doi:10.1111/j.1468-1331.2008.02431.x.

91. Deshmukh-Taskar P, Nicklas TA, Morales M, Yang SJ, Zakeri I, Berenson GS. Tracking of overweight status from childhood to young adulthood: the Bogalusa Heart Study. Eur J Clin Nutr. 2006;60(1):48-57. doi:10.1038/sj.ejcn.1602266.

92. Ascherio A, Munger KL. E Epidemiology of multiple sclerosis: from risk factors to prevention-an update. Semin Neurol. 2016;36(2):103-114. doi:10.1055/s-0036-1579693.

93. Ogden CL, Carroll MD, Kit BK, Flegal KM. Prevalence of childhood and adult obesity in the United States, 2011-2012. JAMA. 2014;311(8):806-814. doi:10.1001/jama.2014.732.

94. Guerrero-Garcia JJ, Carrera-Quintanar L, Lopez-Roa RI, MarquezAguirre AL, Rojas-Mayorquin AE, Ortuno-Sahagun D. Multiple sclerosis and obesity: possible roles of adipokines. Mediators Inflamm. 2016;2016:4036232. doi:10.1155/2016/4036232.

95. Emamgholipour S, Eshaghi SM, Hossein-nezhad A, Mirzaei K, Maghbooli Z, Sahraian MA. Adipocytokine profile, cytokine levels and foxp3 expression in multiple sclerosis: a possible link to susceptibility and clinical course of disease. PLoS One. 2013;8(10):e76555. doi:10.1371/journal.pone.0076555.

96. Salim MA, Eftekharian MM, Taheri $M$, Yousef Alikhani $M$. Determining the IgM and IgG antibody titer against CMV and helicobacter pylori in the serum of multiple sclerosis patients comparing to the control group in Hamadan. Hum Antibodies. 2017;26(1):23-28. doi:10.3233/hab-170317.

97. KarampoorS, Zahednasab H, RamagopalanS, etal. Cytomegalovirus and varicella zoster virus seropositivity of Iranian patients with multiple sclerosis: A population-based study. J Neuroimmunol. 2017;309:4-6. doi:10.1016/j.jneuroim.2017.04.004.

98. Najafi S, Ghane M, Poortahmasebi V, Jazayeri SM, YousefzadehChabok S. Prevalence of cytomegalovirus in patients with multiple sclerosis: a case-control study in northern Iran. Jundishapur J Microbiol. 2016;9(7):e36582. doi:10.5812/jjm.36582.

99. Martinez-Rodriguez JE, Cobo-Calvo A, Villar LM, et al. Adaptive natural killer cell response to cytomegalovirus and disability progression in multiple sclerosis. Mult Scler. 2016;22(6):741-752. doi:10.1177/1352458515601215.

100. Jaruvongvanich V, Sanguankeo A, Jaruvongvanich S, Upala S. Association between Helicobacter pylori infection and multiple sclerosis: A systematic review and meta-analysis. Mult Scler Relat Disord. 2016;7:92-97. doi:10.1016/j.msard.2016.03.013.

101. Yao G, Wang P, Luo XD, Yu TM, Harris RA, Zhang XM. Meta- analysis of association between Helicobacter pylori infection and multiple sclerosis. Neurosci Lett. 2016;620:1-7. doi:10.1016/j. neulet.2016.03.037.

102. Park AM, Omura S, Fujita M, Sato F, Tsunoda I. Helicobacter pylori and gut microbiota in multiple sclerosis versus Alzheimer's disease: 10 pitfalls of microbiome studies. Clinical and Experimental Neuroimmunology. 2017;8(3):215-232. doi:10.1111/cen3.12401.

103. Massa J, O'Reilly EJ, Munger KL, Ascherio A. Caffeine and alcohol intakes have no association with risk of multiple sclerosis. Mult Scler. 2013;19(1):53-58. doi:10.1177/1352458512448108.

104. ZhuT, YeX, ZhangT, et al. Association between alcohol consumption and multiple sclerosis: a meta-analysis of observational studies. Neurol Sci. 2015;36(9):1543-1550. doi:10.1007/s10072-0152326-7.

105. D'Hooghe M B, Haentjens P, Nagels G, De Keyser J. Alcohol, coffee, fish, smoking and disease progression in multiple sclerosis. Eur J Neurol. 2012;19(4):616-624. doi:10.1111/j.14681331.2011.03596.x.

106. Diaz-Cruz C, Chua AS, Malik MT, et al. The effect of alcohol and red wine consumption on clinical and MRI outcomes in multiple sclerosis. Mult Scler Relat Disord. 2017;17:47-53. doi:10.1016/j. msard.2017.06.011.

107. Hedstrom AK, Mowry EM, Gianfrancesco MA, et al. High consumption of coffee is associated with decreased multiple sclerosis risk; results from two independent studies. J Neurol Neurosurg Psychiatry. 2016;87(5):454-460. doi:10.1136/jnnp2015-312176.

108. Madeira MH, Boia R, Ambrosio AF, Santiago AR. Having a coffee break: the impact of caffeine consumption on microglia-mediated inflammation in neurodegenerative diseases. Mediators Inflamm. 2017;2017:4761081. doi:10.1155/2017/4761081.

109. Sieber M. Neuroprotective properties of nicotine. Curr Med Chem. 2012;19(2):292-297.doi:10.2174/092986712803414222.

110. Quik M, O'Leary K, Tanner CM. Nicotine and Parkinson's disease: implications for therapy. Mov Disord. 2008;23(12):1641-1652. doi:10.1002/mds.21900.

111. Quik M, Perez XA, BordiaT. Nicotine as a potential neuroprotective agent for Parkinson's disease. Mov Disord. 2012;27(8):947-957. doi: $10.1002 / \mathrm{mds} .25028$.

112. Lombardo S, Maskos U. Role of the nicotinic acetylcholine receptor in Alzheimer's disease pathology and treatment. Neuropharmacology. 2015;96(Pt $\quad$ B):255-262. doi:10.1016/j. neuropharm.2014.11.018.

113. Naddafi F, Reza Haidari M, Azizi G, Sedaghat R, Mirshafiey A. Novel therapeutic approach by nicotine in experimental model of multiple sclerosis. Innov Clin Neurosci. 2013;10(4):20-25. doi:10.1007/s13760-014-0392-x.

114. Khezri S, Abtahi Froushani SM, Shahmoradi M. Nicotine augments the beneficial effects of mesenchymal stem cell-based therapy in rat model of multiple sclerosis. Immunol Invest. 2018;47(2):113124. doi:10.1080/08820139.2017.1391841. 